\title{
Screening of Indigenous Lactic Acid Bacteria from Raw and Fermented Milk for Probiotic Potential
}

\author{
${ }^{1}$ Aforijiku, S, ${ }^{2}$ Onilude, A. A. and ${ }^{3}$ Wakil, S. M \\ ${ }^{1-3}$ Department of Microbiology \\ University of Ibadan \\ Ibadan, Oyo State, Nigeria
}

\begin{abstract}
Lactic acid bacteria are known to have probiotic attributes which are beneficial to human. This study was embarked upon to screen lactic acid bacteria (LAB) from raw milk and fermented milk (nono) samples for probiotic potential. Some of the assessed probiotic qualities include antimicrobial activities against food-borne pathogens, survival at acidic pH (2.5, 3.0) and bile salt concentrations $(0.3 \%, 0.5 \%, 1.0 \%)$, safety test, and cell surface hydrophobicity assay indicative of epithelial adherence. Four LAB isolates namely Lactobacillus plantarumN17, L. plantarumN24, L. caseiN1 and L. brevisN10 had strong inhibition (10.00 to $15.15 \mathrm{~mm}$ ) against selected food-borne pathogens, survived well at acidic pH and bile salt concentrations during 3 hours of incubation reaching viability of $10^{5}-10^{6} \mathrm{CFU} / \mathrm{mL}$.In addition, they were DNase and Gelatinase negative, and had better(40.0 to $62.0 \%)$ hydrophobicity indicative of epithelial adherence. Lactobacillus plantarumN24 and Lactobacillus caseiN1 were suitable probiotic candidates, and can be used as for food supplements.
\end{abstract}

Key Words: Lactic acid bacteria (LAB), Nono, Probiotic.

\section{INTRODUCTION}

Lactic acid bacteria have been known in the food industries and still in existence till date. Moreover, they are also regarded as fastidious, acid tolerant, micro-aerophilic organisms, and have the ability to produce lactic acid (Rivera- Espinoza and GallardoNavaro, 2010; Brant and Todd, 2014).

Lactic acid bacteria (LAB)can be used as probiotics based on immense benefits. Most of the lactic acid bacteria in most work can be used as protective organisms which can be inoculated in yoghurt (Pérez-Chabela et al., 2008) or embedded with biopolymers (Pérez-Chabela et al., 2012; Pérez-Chabela et al., 2013; Mohammed et al., 2016). Probiotics refer to living microorganisms that has the tendency to exert some biological changes in the gut or gastrointestinal compartment when ingested by man (Masci et al., 2013; Vasiee et al., 2017). These probiotic organisms have the tendency to inhibit pathogens, but should also exhibit some important probiotic potentials. Moreover, some criteria used for regarding an organisms as probiotic bacteria includes tolerance to bile salts, survival at acidic $\mathrm{pH}$, adherence to intestinal mucosa or epithelial cells, and should also be safe ( Mohammed et al., 2016). However, survival of LAB in yoghurt is a challenge to quality product delivery because most LAB cannot withstand the low $\mathrm{pH}$ during yoghurt production and harsh conditions of the gut, hence limiting their probiotic potentials. Therefore, this study was designed to screen and select probiotic LAB starters

\section{MATERIALS AND METHODS}

\subsection{Collection of samples}

Raw milk from cow, goat and traditional fermented milk product (nono samples) were randomly purchased purposively from 'kara' at Bodija in Ibadan, Nigeria. They were brought into the laboratory in sterile bottles for microbiological analysis.

\subsection{Collection of Indicator organisms}

Indicator organisms were obtained from the culture collection unit of Federal Institute of Industrial Research, Oshodi (FIIRO).The organisms are Escherichia coli ATCC 8739, Salmonella tyhimurium ATCC 13311, Bacillus subtilis ATCC 6633, Proteus spp, Shigella flexneri ATCC 29833, Bacillus cereus CMGB 215. 


\subsection{Isolation and characterisation of isolates}

Isolation of lactic acid bacteria was done using pour plate technique. One millilitre of each raw milk samples from goat, cow, and nono were taken aseptically and transferred into separate bottles containing $9.0 \mathrm{~mL}$ of sterile distilled water, and serial dilutions of the milk samples were made. One millilitre of $10^{-6}$ dilutions of the samples was plated into sterile Petri dishes containing MRS agar, and incubated in anaerobic jars at $37^{\circ} \mathrm{C}$ for $48 \mathrm{hr}$. Isolates were sub-cultured and repeated streaking was done to obtain pure cultures (Nikolic et al., 2008). The isolates were characterized using conventional procedures by employing macroscopic, microscopic, physiological and biochemical tests.

\subsection{Screening of isolated LAB for probiotic potential}

The isolated LAB were assessed for probiotic potential which includes:

\subsection{Antimicrobial activities of LAB against food-borne pathogens}

Antimicrobial effects of metabolites of presumptive species of LAB isolates against Escherichia coli ATCC 8739, Salmonella tyhimurium ATCC 13311, Bacillus subtilis ATCC 6633, Proteus spp, Shigella flexneri ATCC 29833, Bacillus cereus CMGB 215earlier stated were determined by Agar diffusion method (Nikolic et al., 2008). The indicator organisms were incubated in nutrient broth at $37^{\circ} \mathrm{C}$ for $24 \mathrm{hr}$, and $100 \mu \mathrm{L}$ (approximately inoculum size of $10^{7} \mathrm{CFU} / \mathrm{mL}$ ) of standardized over night cultures was used. A $50 \mu \mathrm{L}$ of cell free supernatants of cultured MRS broth obtained by centrifugation ( $4000 \mathrm{~g}$ for 15 minutes), was filled in $8 \mathrm{~mm}$ diammeter sealed wells cut on Mueller Hinton agar containing the test organisms. It was stored in the refrigerator for 2 $\mathrm{hr}$, and the inoculated plates were incubated at $37^{\circ} \mathrm{C}$ for $24 \mathrm{hr}$. The diammeter of the inhibition zone was measured with a transparent ruler to the nearest milliliters from the point of inhibition to the end.

\subsection{Tolerance (growth) of $\mathrm{LAB}$ to different concentration of $\mathrm{NaCl}$}

One milliliter of the LAB isolates were inoculated into $10 \mathrm{~mL}$ freshly prepared MRS containing $4.0 \%, 6.0 \%, 8.0 \%$ and $10.0 \%$ $\mathrm{NaCl}$ and their growth were assessed for $48 \mathrm{hr}$. The growth was confirmed on MRS broth by visual reading due to its turbidity (Hoque et al., 2010).

\subsection{Survival of $\mathrm{LAB}$ at acidic $\mathrm{pH}$ under various incubation time}

The LAB isolates were tested for their tolerance to acidic conditions similar to those of the stomach. The acid resistance was examined in MRS broth adjusted with Conc. $\mathrm{HCl}$ to $\mathrm{pH} 2.5,3.0$, and 4.0. Each LAB isolate was inoculated separately $\left(10^{7}\right.$ $\mathrm{CFU} / \mathrm{mL}$ ) in $10 \mathrm{~mL}$ MRS broth at $\mathrm{pH}$ 2.5, 3.0, 4.0. After incubation for 45 minutes, $1 \mathrm{hr}, 2 \mathrm{hr}, 3 \mathrm{hr}$ and $24 \mathrm{hr}$, viable cells of the isolates were confirmed on MRS agar after anaerobic incubation at $37^{\circ} \mathrm{C}$ for $48 \mathrm{hr}$ following the protocol of Klingberg et al. (2005). A period of 45 minutes acclimatization time was used during the start of incubation in this study and was indicated as $0 \mathrm{hr}$.

\subsection{Tolerance to bile salts under various incubation time}

The LAB isolates were tested for their ability to survive different concentration of bile salts. Each LAB isolates were separately inoculated $\left(10^{7} \mathrm{CFU} / \mathrm{mL}\right)$ into $10 \mathrm{~mL}$ MRS broth containing varying concentration of bile salts $(0.3 \%, 0.5 \%, 1.0 \%)$. After exposures to incubation for 45 minutes, $1 \mathrm{hr}, 2 \mathrm{hr}$, and $3 \mathrm{hr}$, viable cells were confirmed on MRS Agar after anaerobic incubation for $48 \mathrm{hr}$ at $37^{\circ} \mathrm{C}$ (Klingberg et al., 2005). A period of 45 minutes acclimatization time was used during the start of incubation in this study.

\subsection{Adherence to intestinal mucosa using hydrophobicity assay}

The protocol of Rosenberg et al. (2013) was used. The LAB isolates were first grown on MRS broth at $37^{\circ} \mathrm{C}$ for $24 \mathrm{hr}$. They were centrifuged at $5000 \mathrm{x}$ g for 15 minutes, pellets was washed twice with phosphate buffer saline (PBS) having pH 7.0, and the optical density was measured at $540 \mathrm{~nm}$. Then, one milliliter of the bacterial suspension was added to $1 \mathrm{~mL}$ of different hydrocarbons (chloroform and xylene) and were vortexed for 30 seconds. After 30 minutes of phase separation, the optical density of aqueous separation was measured again at $540 \mathrm{~nm}$ and was compared with initial value. Hydrophobicity was calculated using the equation:

$\%$ hydrophobicity $=\left(\mathrm{A}_{540 \mathrm{~nm}}\right.$ initial value- $\mathrm{A}_{540 \mathrm{~nm}}$ aqueous solution/A540nm $) \times 100$

\subsection{Antibiotic susceptibility test of LAB}

A total of eight antibiotics discs (Oxoid, England) were used to determine the sensitivity of LAB to antibiotics. They are Ceftazidime $(10 \mu \mathrm{g})$, Cefuroxime $(30 \mu \mathrm{g})$, Gentamycin $(30 \mu \mathrm{g})$, Ceftriaxone $(20 \mu \mathrm{g})$, Ofloxacin $(10 \mu \mathrm{g})$, Erythromycin $(10 \mu \mathrm{g})$, Augmentin $(30 \mu \mathrm{g})$, and Cloxacin $(10 \mu \mathrm{g})$. The bacterial cultures of $18 \mathrm{hr}$ old were inoculated into $10 \mathrm{~mL}$ of normal saline which had being standardized to cell suspension (Mcfarland standard 0.5). The saline containing the cultures were flooded on Mueller- 
Hinton agar plates before introducing the disc with a sterile forceps. The plates was incubated for $48 \mathrm{hr}$ at $37^{\circ} \mathrm{C}$, and the zone of inhibition was measured with a transparent ruler to the nearest milliliters, and compared with the values of susceptibility interpretation break points, and was expressed in terms of resistance and sensitive (Bauer et al., 1996).

\subsection{Safety assessment of LAB based on gelatinase and Dnase production}

\section{Gelatinase production}

Gelatinase production was determined by inoculating $18 \mathrm{hr}$ old cultures of the LAB isolates on plate containing nutrient agar, supplemented with $0.4 \%$ gelatin and was incubated at $37^{\circ} \mathrm{C}$ for $48 \mathrm{hr}$. The incubated plate was flooded with saturated ammonium sulphate solution. The development of clear zones around the spots against the opaque background indicated a positive reaction while absence of clear zones indicated negative result (Gupta and Malik, 2007).

\subsection{DNase production}

DNase agar medium was used to check production of DNase enzyme. A streaked of the LAB cultures were made on the agar and incubated at $30^{\circ} \mathrm{C}$ for $48 \mathrm{hr}$. After incubation, a clear pinkish zone around the colonies against dark-blue background was considered positive for DNase production (Gupta and Malik, 2007).

\section{Statistical analysis}

The experiments was carried out in duplicates, average was recorded, and Analysis of Variance (ANOVA) with Duncan Multiple Range Test for significance at $\mathrm{P} \leq 0.05$ was also used.

\subsection{RESULTS AND DISCUSSION}

Fifty - five presumptive lactic acid bacteria were isolated from raw cow milk, raw goat milk and nono samples. On the basis of the cultural and morphological appearances, all the LAB isolates were small colonies, circular, whitish to creamy in colour, raised with entire edges. They were all Gram positive, short to long rods. On the basis of the identification tests, isolates were identified as Pediococcus acidilactici, Lactobacillus plantarum, Lactobacillus brevis, Lactobacillus fermentum and Lactobacillus casei according to Bergey's Manual of Determinative Bacteriology based on their similarities in characteristics with the organisms (Nikolicet al., 2008).The results was not shown.

\subsection{Screening of LAB for probiotic potential}

\subsection{Antimicrobial activities of LAB against food -borne pathogens}

The antagonistic activities exhibited by LAB isolated from raw milk and nono samples is presented in Table 1. Culture supernatants of the isolates obtained from Lactobacillus plantarum, Lactobacillus casei, Lactobacillus brevis, and Lactobacillus fermentum exhibited varying degrees of inhibitory activity from 7 to $15 \mathrm{~mm}$ against strains of Escherichia coli ATCC8739, Bacillus cereus CMGB215, Proteus spp, Bacillus sublitisATCC6633, Shigella flexneriATCC29833 and Salmonella typhimuriumATCC13311. Lactobacillus plantarumN24, Lactobacillus plantarumN17 and Lactobacillus brevisN10isolated from nono samples had good inhibition against Salmonella typhimuriumATCC13311 with $15.00 \pm 0.00 \mathrm{~mm}, 15.15 \pm 2.76 \mathrm{~mm}$, $15.10 \pm 1.27 \mathrm{~mm}$, respectively and were not significantly different from each other at $\mathrm{P} \leq 0.05$ but slightly different from Lactobacillus caseiN1( $14.00 \pm 0.57 \mathrm{~mm})$. They also had inhibition against Shigella flexneriATCC29833 and E.coliATCC873. About $52.7 \%$ of the LAB metabolites inhibited Proteus spp while 47.2\% showed no inhibition. The results of some LAB are not shown in the table. In a study experimented by Yadesse et al. (2005), they suggested that the antimicrobial potential of LAB could be influenced by the medium they grew in, biochemical properties of the strains used including major factors such as physical and chemical conditions of growth.

However, these compounds produced by LAB lowered the $\mathrm{pH}$ of the medium which could result to strong antagonistic effect against the studied food -borne pathogens or indicator organisms (Krishnendra et al., 2013).

\subsection{Tolerance (growth) of $\mathrm{LAB}$ to various $\mathrm{NaCl}$ concentration}

The result showed that all the studied LAB isolates were able to tolerate 4-10\% $\mathrm{NaCl}$ concentration with different growth rate as shown in Table 2. At $4.0 \% \mathrm{NaCl}$ concentration, normal growth was observed by Lactobacillus plantarum 17 , Lactobacillus plantarum $\mathrm{N} 24$, Lactobacillus brevis $\mathrm{N} 10$ and Lactobacillus casei $\mathrm{N} 1$,of which profuse growth was observed by them at $6.0 \%$ and $8.0 \% \mathrm{NaCl}$ concentration. However, $70.37 \%$ (38) of the LAB tolerated $4.0 \% \mathrm{NaCl}$ concentrations while 29.6\% (16) were not able to grow. Pediocococcus acidilactici did not grow at $4 \%$ but grew at other concentration of NaCl. Moreover, $100 \%$ of the LAB were able to grow at $6 \%, 8 \%$ and $10 \%$ salt concentrations. The presence of this salt will have no adverse effects on the organisms. 
Hoque et al. (2010) reported the tolerance of some Lactobacillus species isolated from fermented milk products to $1-9.0 \% \mathrm{NaCl}$ concentrations as indicated in their experiments. However, results of this experimental studies are similar to the work done by Adebayo-tayo and Onilude (2008), on the tolerance of LAB to $1-6.5 \% \mathrm{NaCl}$.

\subsection{Survival of LAB at acidic $\mathrm{pH}$ under various incubation time}

The results of survival at different acidic $\mathrm{pH}$ are shown in Figures 1 and 2. The LAB isolates were able to grow at $\mathrm{pH} 2.5$ for 45 minutes but Lactobacillus plantarum $\mathrm{N} 24$ exhibited the best survival $\left(3.6 \times 10^{6} \mathrm{CFU} / \mathrm{mL}\right)$. However, all the isolates lost their viability except for Lactobacillus plantarumN17, Lactobacillus plantarum N24, Lactobacillus caseiN1and Lactobacillus brevis $\mathrm{N} 10$ showing viability of $10^{6} \mathrm{CFU} / \mathrm{mL}$ during $2 \mathrm{hr}$ of incubation $\left(1.0 \times 10^{6}, 1.8 \times 10^{6}, 2.8 \times 10^{6}\right.$ and $1.5 \times 10^{6} \mathrm{CFU} / \mathrm{mL}$, respectively). At pH 3.0 as shown in Figure 2, Lactobacillus plantarumN24 and Lactobacillus plantarumN17 survived better reaching viability of $\left(5.5 \times 10^{6} \mathrm{CFU} / \mathrm{mL}\right)$ and $\left(5.6 \times 10^{6} \mathrm{CFU} / \mathrm{mL}\right)$, respectively at 45 mins but Lactobacillus brevis $\mathrm{N} 10$ survived best after $3 \mathrm{hr}$ of incubation.

Moreover, this work reveals that four LAB isolated from fermented milk product (nono samples) were able to survived pH 2.5 and 3.0 for $3 \mathrm{hr}$.. Kabore et al. (2012) also isolated LAB from a fermented milk product, and they were able to survive well at $\mathrm{pH} 2.5$ and above, suggesting it could be intrinsical. Furthermore, LAB strains were shown such as Lactobacillus casei can resist $\mathrm{pH} 3.0$ for $3 \mathrm{hr}$. This could be attributed to the medium they grew in or transfer of acidic genes that can resist low $\mathrm{pH}$ (Mishra and Prasad, 2005; Kumar and Kumar, 2015).

Table 1: Antimicrobial activities of LAB isolates against food-borne pathogens (diameter of zones of inhibition $(\mathbf{m m}))$

Zones of Inhibition (mm)

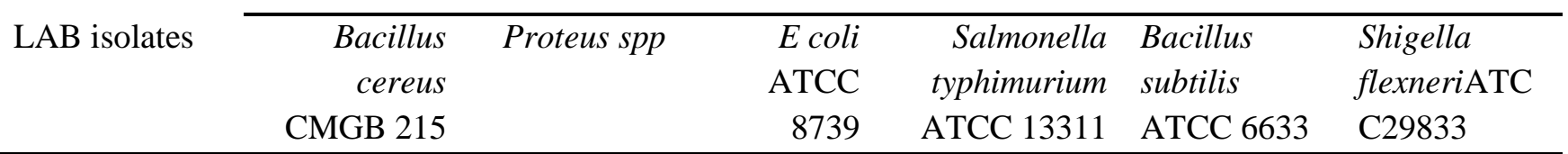

$P$.

acidilacticiG1

$P$.

acidilactici $\mathrm{G} 2$

$P$.

acidilactici $\mathrm{G} 3$

$P$.

acidilactici $\mathrm{G} 4$

$P$.

acidilacticiG5

$P$.

acidilacticiG6

L.

$11.00 \pm 0.00^{\text {def }} \quad 10.00 \pm 0.00^{\text {fgh }} \quad 12.15 \pm 0.1^{\mathrm{b}} \quad 12.00 \pm 0.00^{\text {cd }}$

plantarum $\mathrm{G} 7$

$P$.

acidilactici $\mathrm{G} 8$

$P$.

acidilactici $\mathrm{G} 9$

$P$.

acidilactici $\mathrm{G} 10$

$P$. acidilacticiG11

L. $\quad 8.00 \pm 0.00^{\mathrm{i}} \quad 10.70 \pm 0.5^{\text {ef }} \quad 11.00 \pm 0.00^{\text {def }}$

plantarum $\mathrm{G} 12$

$P$.

acidilacticiC1

$P$.

acidilacticiC2 
International Journal of Advances in Scientific Research and Engineering (ijasre),Vol 5 (6), June-2019

\begin{tabular}{lcccc}
\hline L. & $8.00 \pm 1.41^{\mathrm{i}}$ & $11.00 \pm 0.35^{\mathrm{ef}}$ & & $10.00 \pm 2.62^{\mathrm{f}}$ \\
plantarum $\mathrm{C} 3$ & & & & \\
L. & $10.00 \pm 0.00^{\mathrm{fgh}}$ & $10.00 \pm 1.41^{\mathrm{fgh}}$ & & \\
plantarum $\mathrm{C} 4$ & & & & \\
L. & & $12.00 \pm 0.00^{\mathrm{b}-\mathrm{f}}$ & $10.25 \pm 0.3^{\mathrm{f}}$ & $11.15 \pm 0.07^{\mathrm{de}}$ \\
plantarum $\mathrm{C} 5$ & & & \\
$\begin{array}{l}\text { L. } \\
\text { plantarum } \mathrm{C} 6\end{array}$ & & $11.00 \pm .0 .00^{\mathrm{def}}$ & \\
\hline
\end{tabular}

*Means of duplicates with the same alphabets within a column are not significantly different at $\mathrm{P} \leq 0.05$ using Duncan Multiple Range Test (DMRT) for separation of statistically significant. $8-15 \mathrm{~mm}=$ Good Inhibition, - = No inhibition, low inhibition $=7 \mathrm{~mm}, \mathrm{G}=$ Isolates from goat milk, $\mathrm{C}=$ Isolates from cow milk, $\mathrm{N}=$ Isolates from nono samples, LAB isolates = Lactic acid bacterial isolates

Table 1 (Cont'd):

Zones of Inhibition (mm)

\begin{tabular}{lllllcc} 
& Bacillus & Proteus spp & E coli & Salmonella & Bacillus & Shigella \\
LAB isolates & cereusCMGB2 & & ATCC 8739 & typhimurium & subtilis & flexneriATCC \\
& 15 & & ATCC & ATCC 6633 & 29833 \\
& & & 13311 & & \\
\hline
\end{tabular}

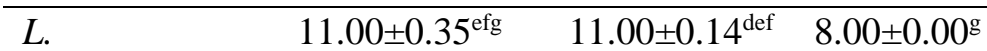

plantarum $\mathrm{C7}$

L. $\quad 11.00 \pm 0.00^{\mathrm{def}}$

plantarum $\mathrm{C} 8$

$L$.

$12.00 \pm 0.57^{\mathrm{c}-\mathrm{f}}$

$10.00 \pm 0.28^{\mathrm{ef}}$

plantarum $\mathrm{C} 9$

$L$.

$10.00 \pm 1.41^{\mathrm{fgh}}$

$10.00 \pm 0.28^{\mathrm{f}}$

$11.00 \pm 2.55^{\text {def }}$

plantarum $\mathrm{C} 10$

$P$.

acidilacticiC11

$L$.

plantarum $\mathrm{C} 12$

$10.00 \pm 0.28^{\text {fgh }} \quad 12.00 \pm 1.34^{\text {b-f }}$

$8.00 \pm 0.42^{\mathrm{g}}$

$10.00 \pm 2.26^{\mathrm{ef}}$

$L$.

$13.00 \pm 1.41^{\mathrm{a}-\mathrm{d}}$

$10.20 \pm 0.28^{\mathrm{ef}}$

$10.00 \pm 1.48^{\mathrm{f}}$

plantarum $\mathrm{C} 13$

L

$12.00 \pm 0.42^{\text {cd }}$

$12.00 \pm 0.00^{\mathrm{b}-\mathrm{f}}$

plantarum $\mathrm{C} 14$

$L$.

$12.00 \pm 0.21^{\text {cde }}$

$10.00 \pm 2.83^{\text {fgh }}$

$11.00 \pm 0.28^{\mathrm{ef}}$

$12.00 \pm 1.41^{\mathrm{cd}}$

$12.00 \pm 1.41^{\mathrm{cd}}$

plantarum $\mathrm{C} 15$

$P$.

acidilacticiC16

$P$.

acidilacticiC17

L.

L. casei $\mathrm{N} 1$

$14.00 \pm 0.28^{\mathrm{ab}}$

$13.00 \pm 2.83^{\mathrm{a}-\mathrm{d}}$

$13.00 \pm 0.21^{\mathrm{ab}}$

$14.00 \pm 0.57^{\mathrm{ab}}$

$14.00 \pm 0.14^{\mathrm{ab}} \quad 14.00 \pm 1.41^{\mathrm{b}}$

plantarum $\mathrm{N} 2$

$L$.

$12.00 \pm 0.21^{\text {cde }}$

$11.00 \pm 1.41^{\text {def }}$

$11.00 \pm 0.00^{\mathrm{ef}}$

$13.00 \pm 0.00^{\mathrm{bc}}$

$13.00 \pm 0.00^{\mathrm{bc}}$

$11.00 \pm 2.83^{\mathrm{def}}$

$12.00 \pm 0.28^{b-f}$

$11.35 \pm 0.49^{\mathrm{c}-}$

plantarum $\mathrm{N} 3$

$L$.

$11.00 \pm 0.00^{\text {def }}$

plantarum $\mathrm{N} 4$

$L$.

$10.10 \pm 0.14^{\mathrm{fgh}}$

$10.35 \pm 0.07^{\text {ef }}$

$14.25 \pm 0.35^{\mathrm{a}}$

$14.00 \pm 0.00^{\mathrm{ab}}$

$13.00 \pm 0.00^{\mathrm{bc}}$

fermentum $\mathrm{N} 5$ 
International Journal of Advances in Scientific Research and Engineering (ijasre),Vol 5 (6), June-2019

\begin{tabular}{|c|c|c|c|c|c|}
\hline $\begin{array}{l}L . \\
\text { plantarum } \mathrm{N} 6\end{array}$ & $15.00 \pm 0.00^{\mathrm{a}}$ & $15.00 \pm 0.00^{\mathrm{a}}$ & $14.00 \pm 0.00^{\mathrm{a}}$ & $14.00 \pm 0.00^{\mathrm{ab}}$ & $14.00 \pm 0.35^{\mathrm{ab}}$ \\
\hline $\begin{array}{l}\text { L. } \\
\text { plantarum } \mathrm{N} 7\end{array}$ & $10.00 \pm 0.00^{\mathrm{fgh}}$ & $10.20 \pm 0.28^{\mathrm{efg}}$ & & $12.00 \pm 0.28^{\mathrm{cd}}$ & \\
\hline
\end{tabular}

*Means with the same alphabets within a column are not significantly different at $\mathrm{P} \leq 0.05$ using Duncan Multiple Range Test (DMRT separation of statistically significant means. Data collected were represented as "Means

Table 1(contd).

\begin{tabular}{|c|c|c|c|c|c|c|}
\hline \multirow[b]{2}{*}{ LAB isolates } & \multicolumn{6}{|c|}{ Zones of Inhibition (mm) } \\
\hline & $\begin{array}{l}\text { Bacillus cereus } \\
\text { CMGB } 215\end{array}$ & Proteus spp & $\begin{array}{l}\text { E.coliATCC } \\
8739\end{array}$ & $\begin{array}{l}\text { Salmonella } \\
\text { typhimurium } \\
\text { ATCC } 13311\end{array}$ & $\begin{array}{l}\text { Bacillus } \\
\text { subtilis ATCC } \\
6633\end{array}$ & $\begin{array}{l}\text { Shigella } \\
\text { flexneriATC } \\
\text { C29833 }\end{array}$ \\
\hline L. brevis $\mathrm{N} 8$ & $11.00 \pm 0.14^{\mathrm{def}}$ & & $14.30 \pm 0.42^{\mathrm{a}}$ & $10.40 \pm 0.85^{\mathrm{ef}}$ & & \\
\hline L. casei $\mathrm{N9}$ & & $12.00 \pm 0.71^{\mathrm{b}-\mathrm{f}}$ & & & $12.00 \pm 0.00^{\mathrm{cd}}$ & \\
\hline L. brevis $\mathrm{N} 10$ & $14.00 \pm 2.83^{\mathrm{ab}}$ & $14.00 \pm 1.41^{\mathrm{a}-\mathrm{c}}$ & $10.20 \pm 0.28^{f}$ & $15.10 \pm 1.27^{\mathrm{a}}$ & $15.00 \pm 0.00^{\mathrm{a}}$ & $8.00 \pm 0.28^{\mathrm{b}}$ \\
\hline L.plantarum $\mathrm{N} 1$ & $12.00 \pm 0.07^{\mathrm{cde}}$ & & $10.75 \pm 0.35^{\text {e-f }}$ & & & \\
\hline LabrevisN12 & $8.00 \pm 0.21^{\mathrm{i}}$ & & $12.20 \pm 1.13^{\mathrm{b}-\mathrm{e}}$ & $11.20 \pm 0.28^{\mathrm{de}}$ & & \\
\hline $\begin{array}{l}\text { L.fermentum } \mathrm{N} 1 \\
3\end{array}$ & & $11.00 \pm 0.49^{\mathrm{def}}$ & $13.00 \pm 4.17^{\mathrm{abc}}$ & & & \\
\hline $\begin{array}{l}L . \\
\text { plantarum } 14\end{array}$ & $9.00 \pm 0.99^{\text {ghi }}$ & $12.00 \pm 1.41^{\mathrm{b}-\mathrm{f}}$ & & $11.00 \pm 0.00^{\mathrm{def}}$ & & \\
\hline L. case $\mathrm{N} 15$ & & & $11.00 \pm 0.42^{\mathrm{def}}$ & $8.15 \pm 0.21^{\mathrm{g}}$ & & \\
\hline $\begin{array}{l}L . \\
\text { plantarum } 16\end{array}$ & $10.00 \pm 0.21^{\mathrm{fgh}}$ & $10.00 \pm 0.21^{\mathrm{e}-\mathrm{f}}$ & & $11.00 \pm 1.41^{\mathrm{def}}$ & & \\
\hline $\begin{array}{l}\text { L. } \\
\text { plantarum N17 } \\
\text { L. } \\
\text { fermentum N18 }\end{array}$ & $13.00 \pm 0.35^{\mathrm{bc}}$ & $14.00 \pm 0.35^{\mathrm{a}-\mathrm{c}}$ & $12.00 \pm 2.69^{\mathrm{b}-\mathrm{e}}$ & $15.15 \pm 2.76^{\mathrm{a}}$ & $14.00 \pm 1.56^{\mathrm{ab}}$ & $15.00 \pm 1.41^{\mathrm{a}}$ \\
\hline $\begin{array}{l}L . \\
\text { plantarum } 19\end{array}$ & & $12.00 \pm 0.21^{\mathrm{b}-\mathrm{f}}$ & $12.00 \pm 2.69^{\mathrm{b}-\mathrm{e}}$ & & & \\
\hline $\begin{array}{l}L . \\
\text { plantarum } 20\end{array}$ & $13.00 \pm 1.34^{\mathrm{bc}}$ & & $13.00 \pm 1.41^{\mathrm{a}-\mathrm{d}}$ & & & \\
\hline $\begin{array}{l}L . \\
\text { brevis } 211\end{array}$ & & & & $11.05 \pm 2.89^{\text {def }}$ & $12.00 \pm 0.92^{\mathrm{cd}}$ & \\
\hline $\begin{array}{l}L . \\
\text { casei } \mathrm{N} 22\end{array}$ & $11.00 \pm 0.28^{\mathrm{def}}$ & $11.00 \pm 0.71^{\mathrm{def}}$ & & $11.20 \pm 0.28^{\mathrm{de}}$ & & \\
\hline $\begin{array}{l}L . \\
\text { plantarum } \mathrm{N} 23\end{array}$ & $9.00 \pm 2.61^{\mathrm{hi}}$ & $10.00 \pm 2.12^{\mathrm{e}-\mathrm{h}}$ & & - & & \\
\hline $\begin{array}{l}L . \\
\text { plantarum } 24\end{array}$ & $14.00 \pm 1.20^{\mathrm{ab}}$ & $12.00 \pm 0.21^{\mathrm{b}-\mathrm{f}}$ & $14.00 \pm 0.28^{\mathrm{ab}}$ & $15.00 \pm 0.00^{\mathrm{a}}$ & $12.00 \pm 0.49^{\mathrm{cd}}$ & $12.00 \pm 0.00^{\mathrm{c}}$ \\
\hline $\begin{array}{l}L . \\
\text { plantarum } 25\end{array}$ & & $8.00 \pm 0.14^{\mathrm{gh}}$ & $8.00 \pm 1.56^{\mathrm{g}}$ & $9.80 \pm 0.57^{\mathrm{f}}$ & & \\
\hline $\begin{array}{l}\text { L. } \\
\text { plantarumN26 }\end{array}$ & & $8.00 \pm 0.99^{\mathrm{h}}$ & & $10.05 \pm 0.07^{\mathrm{ef}}$ & $10.00 \pm 1.41^{\mathrm{f}}$ & \\
\hline
\end{tabular}

Means with the same alphabets within a column are not significantly different at $\mathrm{P} \leq 0.05$ using Duncan Multiple Range (DMRT) for separation of statistically significant means. Data collected were represented as "Means of duplicates \pm Stan 
International Journal of Advances in Scientific Research and Engineering (ijasre),Vol 5 (6), June-2019

Deviation (SD)" only. 8-15 mm = Good Inhibition, $-=$ No inhibition, $7 \mathrm{~mm}=$ low inhibition, LAB isolates= Lactic acid bact isolates

Table 2: Tolerance of $\mathrm{LAB}$ to various concentrations of $\mathrm{NaCl}$

\begin{tabular}{|c|c|c|c|c|}
\hline \multirow[t]{2}{*}{ Isolates } & \multicolumn{4}{|c|}{$\mathrm{NaCl}$ concentrations $(\%)$} \\
\hline & 4.0 & 6.0 & 8.0 & 10.0 \\
\hline P. acidilacticiG1 & - & +++ & +++ & ++ \\
\hline P. acidilactici $\mathrm{G} 2$ & - & +++ & +++ & ++ \\
\hline P. acidilacticiG3 & - & +++ & +++ & ++ \\
\hline P. acidilacticiG4 & - & +++ & ++ & ++ \\
\hline P. acidilacticiG5 & - & ++ & ++ & ++ \\
\hline P. acidilacticiG6 & - & ++ & ++ & ++ \\
\hline L. plantarum $\mathrm{G} 7$ & - & ++ & ++ & + \\
\hline P. acidilacticiG8 & - & ++ & ++ & + \\
\hline P. acidilacticiG9 & - & ++ & ++ & + \\
\hline P. acidilacticiG10 & - & ++ & ++ & ++ \\
\hline P. acidilacticiG11 & - & +++ & +++ & +++ \\
\hline L. plantarum $\mathrm{G} 12$ & ++ & ++ & ++ & ++ \\
\hline P. acidilacticiC1 & - & ++ & +++ & ++ \\
\hline P. acidilacticiC2 & _ & ++ & ++ & ++ \\
\hline L. plantarum $\mathrm{C} 3$ & ++ & ++ & ++ & ++ \\
\hline L. plantarum $\mathrm{C} 4$ & ++ & +++ & ++ & ++ \\
\hline L. plantarum $\mathrm{C} 5$ & ++ & ++ & ++ & ++ \\
\hline L. plantarum $\mathrm{C6}$ & ++ & ++ & ++ & ++ \\
\hline L. plantarum $\mathrm{C} 7$ & ++ & +++ & ++ & ++ \\
\hline L. plantarum $\mathrm{C} 8$ & ++ & +++ & ++ & ++ \\
\hline L. plantarum C9 & ++ & ++ & ++ & ++ \\
\hline L. plantarum $\mathrm{C} 10$ & ++ & ++ & ++ & ++ \\
\hline P. acidilacticiC11 & - & ++ & ++ & ++ \\
\hline L. plantarum $\mathrm{C} 12$ & ++ & ++ & ++ & ++ \\
\hline L. plantarum $\mathrm{C} 13$ & ++ & ++ & +++ & ++ \\
\hline L. plantarum $\mathrm{C} 14$ & ++ & ++ & ++ & + \\
\hline L. plantarum $\mathrm{C} 15$ & ++ & ++ & ++ & ++ \\
\hline P. acidilacticiC16 & - & ++ & ++ & ++ \\
\hline P. acidilacticiC17 & - & ++ & ++ & ++ \\
\hline L. case $\mathrm{N} 1$ & ++ & +++ & +++ & +++ \\
\hline L. plantarum $\mathrm{N} 2$ & ++ & ++ & ++ & + \\
\hline L. plantarum $\mathrm{N} 3$ & ++ & ++ & + & + \\
\hline
\end{tabular}

'+++ Profuse growth, ++ normal , + less growth, - no growth 
International Journal of Advances in Scientific Research and Engineering (ijasre),Vol 5 (6), June-2019

Table 2 contd.

\begin{tabular}{|c|c|c|c|c|}
\hline \multirow[t]{2}{*}{ Isolates } & \multicolumn{4}{|c|}{$\mathrm{NaCl}$ concentration $(\%)$} \\
\hline & 4.0 & 6.0 & 8.0 & 10.0 \\
\hline L. plantarum $\mathrm{N} 4$ & ++ & +++ & + & + \\
\hline L. fermentum $\mathrm{N} 5$ & + & +++ & +++ & ++ \\
\hline L. plantarum $\mathrm{N} 6$ & + & ++ & ++ & ++ \\
\hline L. plantarum $\mathrm{N} 7$ & + & ++ & + & + \\
\hline L. brevis $\mathrm{N} 8$ & ++ & ++ & ++ & + \\
\hline L. casei $\mathrm{N} 9$ & + & ++ & ++ & ++ \\
\hline L.brevis $\mathrm{N} 10$ & ++ & +++ & +++ & +++ \\
\hline L. plantarum $\mathrm{N} 11$ & + & ++ & ++ & ++ \\
\hline L. brevis $\mathrm{N} 12$ & ++ & ++ & ++ & + \\
\hline L. fermentum $\mathrm{N} 13$ & + & ++ & ++ & + \\
\hline L. plantarum $\mathrm{N} 14$ & + & ++ & ++ & + \\
\hline L. case $\mathrm{iN} 15$ & + & ++ & ++ & ++ \\
\hline L. plantarum $\mathrm{N} 16$ & ++ & ++ & ++ & ++ \\
\hline L. plantarum $\mathrm{N} 17$ & ++ & +++ & +++ & +++ \\
\hline L. fermentum $\mathrm{N} 18$ & ++ & ++ & ++ & ++ \\
\hline L. plantarum N19 & ++ & ++ & ++ & ++ \\
\hline L.plantarum 20 & + & ++ & ++ & ++ \\
\hline L. brevis $\mathrm{N} 21$ & ++ & ++ & ++ & ++ \\
\hline L. casei $\mathrm{N} 22$ & ++ & ++ & ++ & + \\
\hline L. plantarum $\mathrm{N} 23$ & ++ & ++ & ++ & ++ \\
\hline L. plantarum $\mathrm{N} 24$ & ++ & +++ & +++ & ++ \\
\hline L. plantarum $\mathrm{N} 25$ & ++ & ++ & ++ & + \\
\hline L. plantarumN26 & ++ & ++ & + & + \\
\hline
\end{tabular}

'+++ Profuse growth, ++ normal growth, + less growth

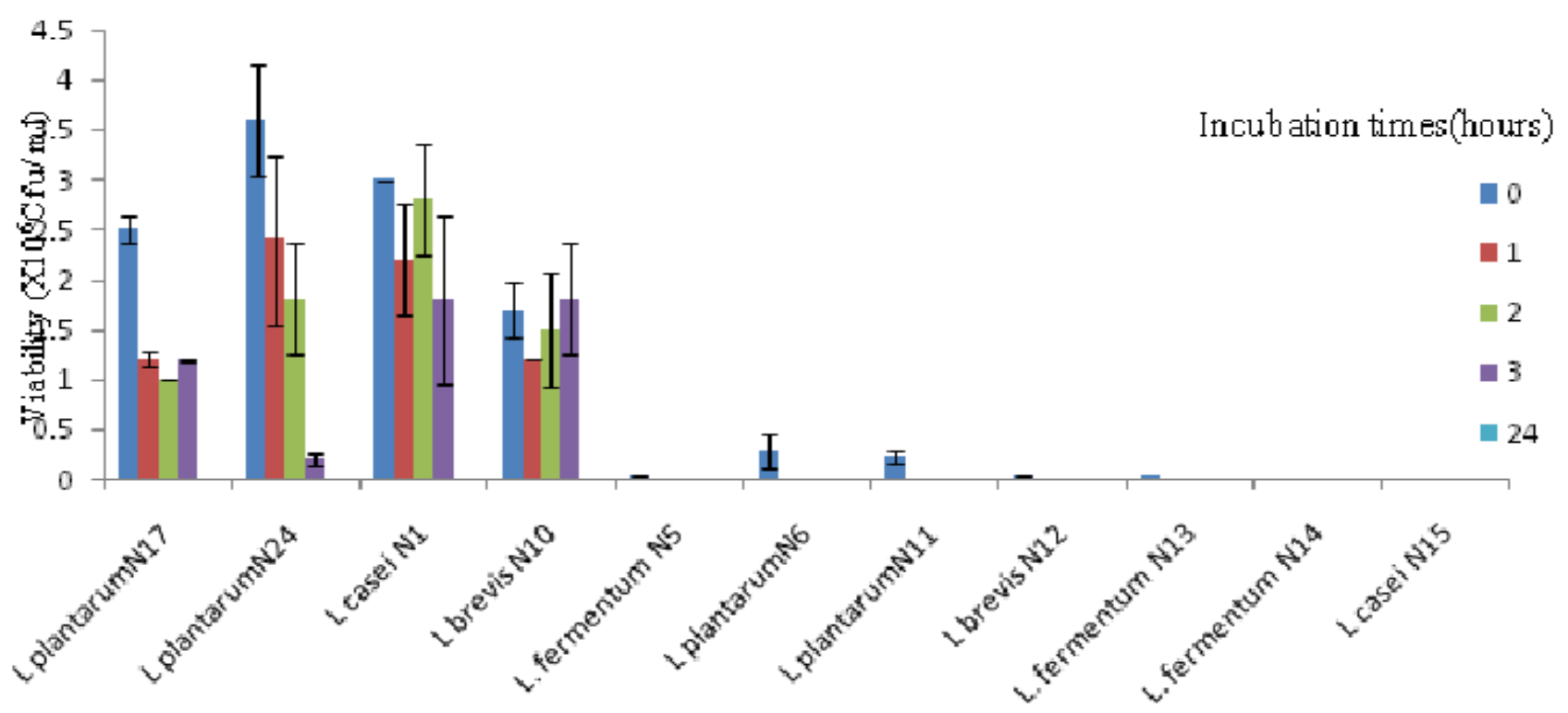

LAB

Figure 1: Survival of LAB at acidic $\mathrm{pH}(\mathrm{pH} 2.5)$ under various incubation times $\left(\mathrm{X10}^{6} \mathrm{CFU} / \mathrm{mL}\right)$. Error bars indicate standard deviations. $0 \mathrm{hr}$ indicates the first ( start) incubation time in this study $=45$ minutes, inoculum size $=10^{7} \mathrm{CFU} / \mathrm{mL}$ 


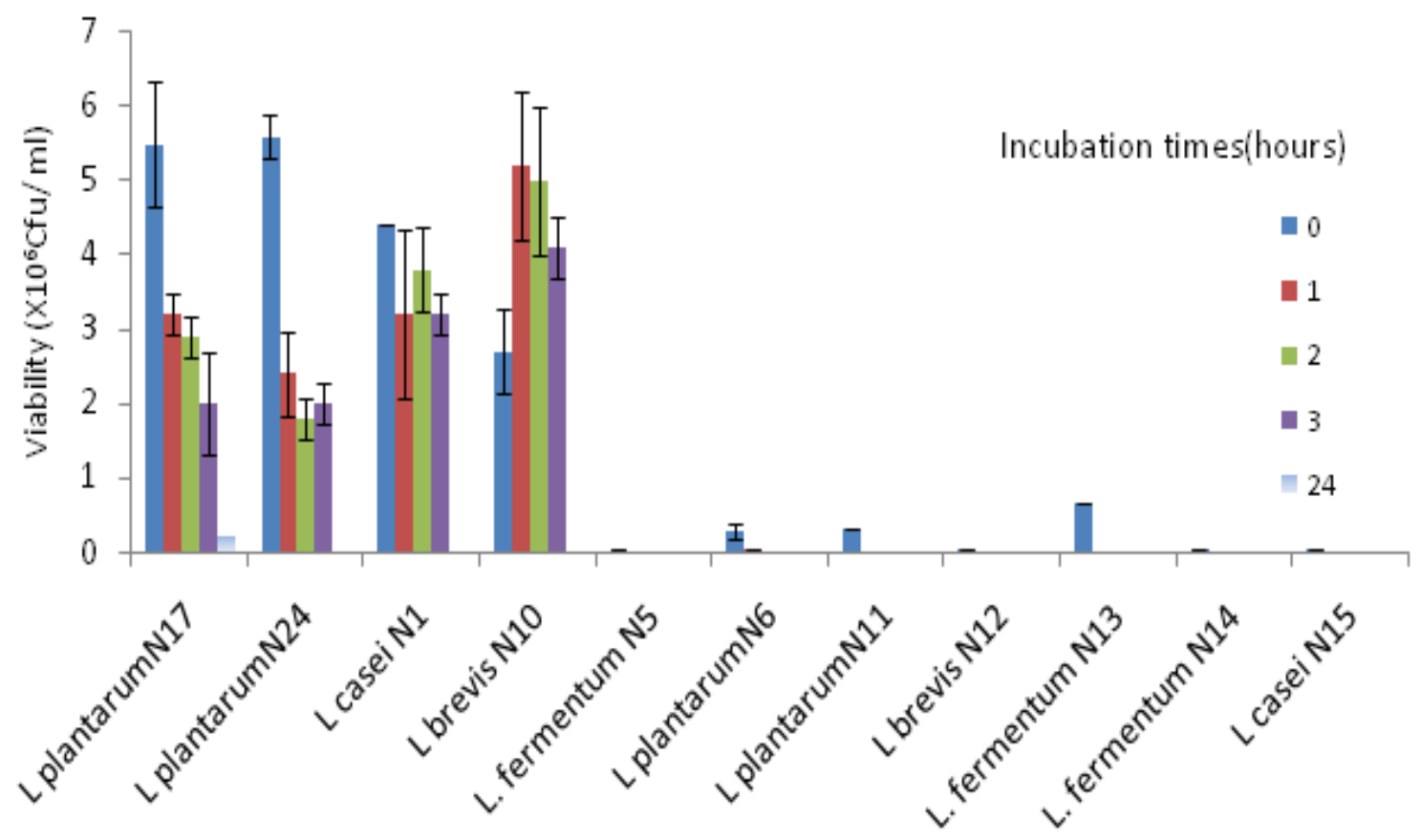

LAB

Figure 2: Survival of $\mathrm{LAB}$ at acidic $\mathrm{pH}(\mathrm{pH} 3.0)$ under various incubation time $\left(\mathrm{X10}^{6} \mathrm{CFU} / \mathrm{ml}\right)$. Error bars indicate standard deviations. $0 \mathrm{hr}$ indicates the first(start) incubation time in this study $=45$ minutes, inoculum size $=10^{7} \mathrm{CFU} / \mathrm{mL}$

\subsection{Tolerance of $\mathrm{LAB}$ to bile salts under various incubation time (CFU/mL).}

Of all the studied $\mathrm{LAB}$, four survived effectively at all the three tested bile salt concentrations $(0.3 \%, 0.5 \%, 1.0 \%)$ as shown in Tables 3, 4 and 5 in which the initial inoculum size was $10^{7} \mathrm{CFU} / \mathrm{mL}$. At $0.3 \%$ bile concentration as shown in Table 3, the studied $\mathrm{LAB}$ showed good survival rate but the level of significance varied and ranged between $0.10-4.0 \times 10^{6} \mathrm{CFU} / \mathrm{mL}$. Lactobacillus plantarum $\mathrm{N} 17$, Lactobacillus brevis $\mathrm{N} 10$, Lactobacillus plantarum $\mathrm{N} 24$, and Lactobacillus case $\mathrm{N} 1$ were seen as the best during (45 minutes) reaching viability of $10^{6} \mathrm{CFU} / \mathrm{mL}(3.5 \pm 1.55,3.0 \pm 1.55,4.0 \pm 0.14,3.00 \pm 1.13 \mathrm{CFU} / \mathrm{mL})$, respectively but were not significantly different from each other at $\mathrm{P} \leq 0.05$. They also survived 2 hrs of incubation. At $0.5 \%$ and $1.0 \%$ bile concentration as shown in Tables 4 and 5, only the four above isolates reached viability of $10^{6} \mathrm{CFU} / \mathrm{mL}$ at $3 \mathrm{hrs}$ of incubation. Generally, there was a slight decrease in the survival rate of the studied LAB as the bile concentration increased but four were able to grow effectively reaching viability of $10^{5}-10^{6} \mathrm{CFU} / \mathrm{mL}$ except for the bile sensitive LAB which lost viability during one hour of incubation at $0.5 \%$ and $1.0 \%$ bile concentration. However, similar work were reported in an in vitro study of Lactobacillus strains which deconjugated the conjugated bile salts due to presence of bile salt hydrolases in the systems. This is attributed to presence of some enzymes that can convert the salts to less toxic substance. Furthermore, most probiotic bacteria were reported to survive and remain viable in MRS medium having above $0.5 \%$ conjugated bile salts (Noruga et al., 2006). Therefore, tolerance to bile salts had being used as a criteria or prerequisite for colonization and metabolic activity of bacteria in the intestine of most host. This present study also revealed that the organisms were able to tolerate at least $0.3 \%$ bile salts which is regarded as the concentration in human.

\subsection{Adherence of LAB to intestinal mucosa using Microbial adherence to hydrocarbons (hydrophobicity assay)}

Table 6 shows the adherence of LAB to hydrocarbons and there was a significant different $(\mathrm{P}<0.05)$ of hydrophobicity of the LAB to hydrocarbons. The percentage hydrophobicity for the tested LAB ranged between $20.8 \pm 0.56$ to $62.0 \pm 0.99 \%$. Lactobacillus case $i \mathrm{~N} 1$ had the highest value of $62.0 \pm 0.99 \%$ towards chloroform which was significantly different $(\mathrm{P}<0.05)$ from that of Lactobacillus plantarumN17 (45.8 $\pm 4.80 \%)$ and Lactobacillus brevisN10 $(40.3 \pm 0.00 \%)$. The least value $(20.8 \pm 0.26 \%)$ was observed by Lactobacillus fermentumN13. Lactobacillus plantarumN24, Lactobacillus brevisN10, Lactobacillus plantarumN17 and Lactobacillus caseiN1 showed above $40 \%$ towards the two solvents (chloroform and xylene) used in this study. 
In xylene, the percentage hydrophobicity ranged between $23.0-53.2 \%$. The highest adherence (53.2 $\pm 0.99 \%)$ was also observed by Lactobacillus caseiN1 which was significantly different from Lactobacillus plantarum 24 (40.7 $\pm 2.68 \%)$ and Lactobacillus plantarum $\mathrm{N} 17$ (40.7 $\pm 2.68 \%$ ). The least adherence was observed by Lactobacillus fermentum 5 showing $23.0 \pm 0.42 \%$.

Microbial adhesion to hydrocarbon has been widely used to determine the cell surface hydrophobicity as adherence to intestinal mucosa or epithelial cells. Orlowaski and Bieleck (2006) suggested that such protocol could determined cell surface hydrophobicity. Lactobacillus species had above $40 \%$ cell surface hydrophobicity towards chloroform and xylene indicating they are strong electron donors with good potential. Kos et al. (2003) reported that Lactobacillus plantanum had maximum cell surface hydrophobicity towards solvents like chloroform and xylene. Other studied LAB had lower percentage $(\leq 40 \%)$ indicating that they are not good probiotics or cannot adhere to intestinal mucosa due to lack of electrostatic interactions. Martin et al. (2005) also reported that some LAB could have low affinity towards chloroform and xylene due to the absence of electrostatic interactions. However, surface hydrophobicity was determined in this study to test for possible correlation between the physiochemical property of LAB and the ability to adhere to the intestinal muscosa. This could also depends on the strain of the microorganisms, surface charge of the bacteria cell and ability of the LAB to express the some associated proteins (Nwanyanwu et al., 2012).

\subsection{Safety assessment ofLAB based on Gelatinase and DNase production.}

Th studied LAB were negative to both gelatinase and DNaseproduction at incubation period of 48 hrs suggesting that they are safe and lack the enzymes indicating. Therefore, they are good probiotic candidates. This is similar to the work of Hasegawa et al. (2010) who reported that probiotic organisms should not produce these enzymes because it can serve as substrates for pathogens and evade the immune systems.

\subsection{Antibiotic susceptibility of LAB}

Table 8 shows the antibiotic susceptibility of the LAB strains. LAB were sensitive to Ceftazidime, Cefuroxime, Gentamycin, Geftriaxone, Ofloxacin, Erythromycin, Augmentin, Cloxacin except Lactobacillus fermentumN5 which was resistance to the some of the antibiotics. Lactobacillus plantarumN6 and Lactobacillus plantarumN11 were also resistant to Augmentin and Cloxacin, respectively but sensitive to other antibiotics. This work reveals that most of the lactic acid bacteria including LactobacilluscaseiN1 and Lactobacillus plantarumN17were sensitive to all the antibiotics which could be intrinsical. This also shows that they lack resistant traits which prevent them from transferring resistant genes to pathogenic organisms. Handa and Sharma (2016) reported that Lactobacillus plantarum were sensitive to majority of the studied antibiotics suggesting such LAB are of advantage, especiallyin the case of transfer of resistance genes to pathogenic organisms.

\section{Table 3: Tolerance of LAB to bile salts $(0.3 \%)$ under various incubation time $\quad\left(\mathrm{X} 10^{6} \mathrm{CFU} / \mathrm{mL}\right)$}

Incubation time (hr)

\begin{tabular}{lllll} 
Isolates & $0(45$ minutes $)$ & 1 & 2 & 3 \\
\hline L. plantarum $\mathrm{N} 17$ & $3.5 \pm 0.84^{\mathrm{a}^{* * *}}$ & $2.8 \pm 0.84^{\mathrm{a}}$ & $2.5 \pm 0.70^{\mathrm{a}}$ & $1.70 \pm 0.42^{\mathrm{a}}$ \\
L. plantarum $\mathrm{N} 24$ & $4.0 \pm 0.14^{\mathrm{a}}$ & $3.1 \pm 0.70^{\mathrm{a}}$ & $1.8 \pm 0.42^{\mathrm{a}}$ & $1.00 \pm 0.00^{\mathrm{a}}$ \\
L. casei $\mathrm{N} 1$ & $3.00 \pm 1.13^{\mathrm{a}}$ & $2.4 \pm 0.99^{\mathrm{a}}$ & $1.6 \pm 0.56^{\mathrm{a}}$ & $1.2 \pm 0.28^{\mathrm{a}}$ \\
L. fermentum $\mathrm{N} 5$ & $0.35 \pm 0.04^{\mathrm{b}}$ & $0.10 \pm 0.00^{\mathrm{b}}$ & - & - \\
L. plantarum $\mathrm{N} 6$ & $0.30 \pm 0.05^{\mathrm{b}}$ & - & - & - \\
L. plantarum $\mathrm{N} 11$ & $0.28 \pm 0.05^{\mathrm{b}}$ & - & - & - \\
L. brevis $\mathrm{N} 12$ & $0.18 \pm 0.02^{\mathrm{b}}$ & $0.13 \pm 0.00^{\mathrm{b}}$ & & - \\
L. fermentum $\mathrm{N} 13$ & $0.18 \pm 0.08^{\mathrm{b}}$ & - & - & - \\
L. plantarum $\mathrm{N} 14$ & $0.20 \pm 0.11^{\mathrm{b}}$ & - & - & - \\
L. casei $\mathrm{N} 15$ & $0.27 \pm 0.00^{\mathrm{b}}$ & - & - & $0.20 \pm 0.04^{\mathrm{b}}$ \\
L. brevis $\mathrm{N} 10$ & $3.0 \pm 1.55^{\mathrm{a}}$ & $2.5 \pm 0.14^{\mathrm{a}}$ & $2.8 \pm 0.84^{\mathrm{a}}$ & \\
\hline
\end{tabular}

**Means with the same alphabets within a column are not significantly different at $\mathrm{P} \leq 0.05$ using Duncan Multiple Range Test (DMRT) for separation of statistically significant means. Data collected were represented as "Means of duplicates \pm 
International Journal of Advances in Scientific Research and Engineering (ijasre),Vol 5 (6), June-2019

Standard Deviation (SD)"VValues are in CFU $/ \mathrm{mL},-=$ not viable, inoculum size $=10^{7} \mathrm{CFU} / \mathrm{mL}, 45$ minutes was used as the first incubation time (start) in this study. Zero point (0.) indicates $10^{5} \mathrm{CFU} / \mathrm{mL}$

Table 4: Tolerance of LAB to bile salts $(0.5 \%)$ under various incubation time $\left(\mathrm{X10}^{6} \mathrm{CFU} / \mathrm{mL}\right)$

Incubation time (hr)

\begin{tabular}{lllll} 
Isolates & $0(45$ minutes $)$ & 1 & 2 & 3 \\
\hline L. plantarum $\mathrm{N} 17$ & $3.0 \pm 1.27^{\mathrm{a}^{* *}}$ & $2.4 \pm 1.13^{\mathrm{a}}$ & $1.8 \pm 0.42^{\mathrm{a}}$ & $1.4 \pm 0.14^{\mathrm{a}}$ \\
L. plantarum $\mathrm{N} 24$ & $3.1 \pm 1.13^{\mathrm{a}}$ & $2.5 \pm 0.42^{\mathrm{a}}$ & $2.4 \pm 0.42^{\mathrm{a}}$ & $1.0 \pm 0.00^{\mathrm{b}}$ \\
L. case $\mathrm{N} 1$ & $2.5 \pm 0.56^{\mathrm{a}}$ & $2.0 \pm 0.70^{\mathrm{a}}$ & $1.4 \pm 0.42^{\mathrm{a}}$ & $1.4 \pm 0.00^{\mathrm{b}}$ \\
L. fermentum $\mathrm{N} 5$ & $0.30 \pm 0.00^{\mathrm{b}}$ & - & - & - \\
L. plantarum $\mathrm{N} 6$ & $0.28 \pm 0.02^{\mathrm{b}}$ & - & - & - \\
L. plantarum $\mathrm{N} 11$ & $0.16 \pm 0.00^{\mathrm{b}}$ & - & - & - \\
L. brevis $\mathrm{N} 12$ & $0.13 \pm 0.02^{\mathrm{b}}$ & - & - & - \\
L. fermentum $\mathrm{N} 13$ & $0.17 \pm 0.07^{\mathrm{b}}$ & - & - & - \\
L. plantarum $\mathrm{N} 14$ & $0.13 \pm 0.02^{\mathrm{b}}$ & - & - & - \\
L. casei $\mathrm{N} 15$ & $0.20 \pm 0.00^{\mathrm{b}}$ & - & - & - \\
L. brevis $\mathrm{N} 10$ & $2.60 \pm 1.13^{\mathrm{a}}$ & $2.4 \pm 0.14^{\mathrm{a}}$ & $0.2 \pm 0.07^{\mathrm{b}}$ & $0.14 \pm 0.05^{\mathrm{c}}$ \\
\hline
\end{tabular}

**Means with the same alphabets within a column are not significantly different at $\mathrm{P} \leq 0.05$ using Duncan Multiple Range Test (DMRT) for separation of statistically significant means. Data collected were represented as "Means of duplicates \pm Standard Deviation .Values are in CFU $/ \mathrm{mL},-=$ not viable, inoculum size $=10^{7} \mathrm{CFU} / \mathrm{mL}, 45$ minutes was used as the first incubation time (start) in this study.

Table 5: Tolerance of LAB to bile salts $(1.0 \%)$ under various incubation time $\left(\mathrm{X10}^{6} \mathrm{CFU} / \mathrm{mL}\right)$

Incubation time (hr)

\begin{tabular}{lllll} 
Isolates & $0(45$ minutes $)$ & 1 & 2 & 3 \\
\hline L. plantarum $\mathrm{N} 17$ & $2.8 \pm 0.99^{\mathrm{a}^{* *}}$ & $2.0 \pm 0.42^{\mathrm{a}}$ & $1.2 \pm 0.28^{\mathrm{a}}$ & $1.2 \pm 0.00^{\mathrm{a}}$ \\
L. plantarum $\mathrm{N} 24$ & $2.2 \pm 0.56^{\mathrm{a}}$ & $1.4 \pm 0.42^{\mathrm{b}}$ & $1.0 \pm 0.00^{\mathrm{a}}$ & $1.0 \pm 0.00^{\mathrm{a}}$ \\
L. casei $\mathrm{N} 1$ & $2.0 \pm 0.70^{\mathrm{a}}$ & $1.6 \pm 0.28^{\mathrm{ab}}$ & $1.2 \pm 0.28^{\mathrm{a}}$ & $1.8 \pm 0.56^{\mathrm{a}}$ \\
L. fermentum $\mathrm{N} 5$ & $0.28 \pm 0.01^{\mathrm{b}}$ & - & - & - \\
L. plantarum $\mathrm{N} 6$ & $0.18 \pm 0.01^{\mathrm{b}}$ & - & - & - \\
L. plantarum $\mathrm{N} 11$ & $0.12 \pm 0.02^{\mathrm{b}}$ & - & - & - \\
L. brevis $\mathrm{N} 12$ & $0.13 \pm 0.00^{\mathrm{b}}$ & - & - & - \\
L. fermentum $\mathrm{N} 13$ & $0.12 \pm 0.02^{\mathrm{b}}$ & - & - & - \\
L. plantarum $\mathrm{N} 14$ & $0.14 \pm 0.01^{\mathrm{b}}$ & - & - & - \\
L. casei $\mathrm{N} 15$ & $0.10 \pm 0.00^{\mathrm{b}}$ & - & - & - \\
L. brevis $\mathrm{N} 10$ & $2.0 \pm 0.99^{\mathrm{a}}$ & $1.4 \pm 0.14^{\mathrm{b}}$ & $0.12 \pm 0.02^{\mathrm{b}}$ & $0.12 \pm 0.02^{\mathrm{b}}$ \\
\hline
\end{tabular}

**Means with the same alphabets within a column are not significantly different at $\mathrm{P} \leq 0.05$ using Duncan Multiple Range Test (DMRT) for separation of statistically significant means. Data collected were represented as "Means of duplicates \pm Standard Deviation (SD)". Values are in CFU $/ \mathrm{mL},-=$ not viable, inoculum size $=10^{7} \mathrm{CFU} / \mathrm{mL}, 45$ minutes was used as the first incubation time (start) in this study. Zero point (0.) indicates $10^{5} \mathrm{CFU} / \mathrm{mL}$ 
International Journal of Advances in Scientific Research and Engineering (ijasre),Vol 5 (6), June-2019

Table 6: Adherence of LAB to intestinal mucosa using hydrophobicity assay

\begin{tabular}{lll}
\hline & \multicolumn{2}{c}{ Hydrophobicity $(\%)$} \\
Isolates & Chloroform $(\mathrm{mL})$ & Xylene $(\mathrm{mL})$ \\
\hline L. plantarum $\mathrm{N} 17$ & $45.8 \pm 4.80^{\mathrm{b} *}$ & $40.7 \pm 2.68^{\mathrm{bc}}$ \\
L. plantarum $\mathrm{N} 24$ & $40.3 \pm 0.00^{\mathrm{bc}}$ & $45.8 \pm 2.12^{\mathrm{b}}$ \\
L. casei $\mathrm{N} 1$ & $62.0 \pm 0.99^{\mathrm{a}}$ & $53.2 \pm 0.99^{\mathrm{a}}$ \\
L. fermentum $\mathrm{N} 5$ & $32.5 \pm 1.41^{\mathrm{de}}$ & $23.0 \pm 0.42^{\mathrm{f}}$ \\
L. plantarum $\mathrm{N} 6$ & $28.5 \pm 1.13^{\mathrm{ef}}$ & $30.7 \pm 2.54^{\mathrm{e}}$ \\
L. plantarum $\mathrm{N} 11$ & $35.0 \pm 1.98^{\mathrm{cde}}$ & $38.5 \pm 0.99^{\mathrm{cd}}$ \\
L. brevis $\mathrm{N} 12$ & $38.7 \pm 0.14^{\mathrm{cd}}$ & $28.5 \pm 1.27^{\mathrm{ef}}$ \\
L. fermentum $\mathrm{N} 13$ & $20.8 \pm 0.56^{\mathrm{g}}$ & $35 \pm 2.26^{\text {cde }}$ \\
L. plantarum $\mathrm{N} 14$ & $25.0 \pm 2.54^{\mathrm{fg}}$ & $32.5 \pm 2.40^{\mathrm{de}}$ \\
L. case $\mathrm{N} 15$ & $22.0 \pm 0.00^{\mathrm{fg}}$ & $31.0 \pm 7.77^{\mathrm{e}}$ \\
L.brevis $\mathrm{N} 10$ & $40.0 \pm 7.49^{\mathrm{bc}}$ & $41.0 \pm 0.14^{\mathrm{bc}}$ \\
\hline
\end{tabular}

*Means with the same alphabets within a column are not significantly different at $\mathrm{P} \leq 0.05$ using Duncan Multiple Range Test (DMRT) for separation of statistically significant means. Data collected were represented as "Means of duplicates \pm Standard Deviation (SD)"

$\geq 40 \%=$ hydrophobic or adherence, less than $40 \%=$ not adhering

Table 7: Safety assessment of LAB based on Gelatinase and DNase production.

\begin{tabular}{|c|c|c|}
\hline \multirow[b]{2}{*}{ Isolates } & \multicolumn{2}{|c|}{ Safety parameters } \\
\hline & $\begin{array}{l}\text { Gelatinase } \\
\text { production }\end{array}$ & DNase production \\
\hline L. plantarum $\mathrm{N} 17$ & - & - \\
\hline L. plantarum $\mathrm{N} 24$ & - & _ \\
\hline L. casei $\mathrm{N} 1$ & - & _- \\
\hline L. fermentum $\mathrm{N} 5$ & - & - \\
\hline L. plantarum $\mathrm{N} 6$ & _ & _ \\
\hline L. plantarum $\mathrm{N} 11$ & - & - \\
\hline L. brevis $\mathrm{N} 12$ & - & _ \\
\hline L. fermentum $\mathrm{N} 13$ & - & - \\
\hline L. plantarum $\mathrm{N} 14$ & - & _- \\
\hline L. casei $\mathrm{N} 15$ & - & - \\
\hline L. brevis $\mathrm{N} 10$ & . & 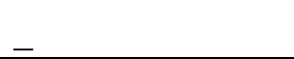 \\
\hline
\end{tabular}

- = negative 
Table 8: Antibiotics susceptibility of LAB

\begin{tabular}{|c|c|c|c|c|c|c|c|c|}
\hline$\varangle$ & 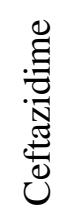 & 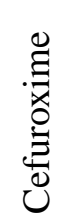 & 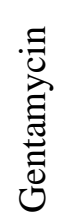 & 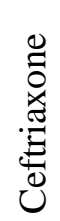 & 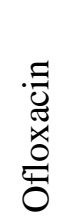 & 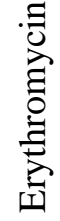 & 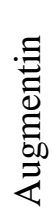 & 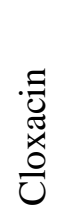 \\
\hline
\end{tabular}

$\begin{array}{llllllllll}\text { L. } & \mathrm{S} & \mathrm{S} & \mathrm{S} & \mathrm{S} & \mathrm{S} & \mathrm{S} & \mathrm{S} & \mathrm{S}\end{array}$

plantarum 17

$L$. plantarum $\mathrm{N} 24$

$\mathrm{S} \quad \mathrm{S} \quad \mathrm{S}$

S

S

S S

S

L. case $i \mathrm{~N} 1$

S S

S

S

S

S

S

S

L. fermentum $\mathrm{N} 5$

S

$\mathrm{R}$

S

S R

$\mathrm{R}$

$\mathrm{R}$

L. plantarum N6

S

S

S

S

S

$\mathrm{R}$

S

$L$.

plantarum N11

S S S

S

S

S

S

R

$\begin{array}{lllllllll}\text { L. brevis } 12 & \mathrm{~S} & \mathrm{~S} & \mathrm{~S} & \mathrm{~S} & \mathrm{~S} & \mathrm{~S} & \mathrm{~S} & \mathrm{~S} \\ \text { L. } & \mathrm{S} & \mathrm{S} & \mathrm{S} & \mathrm{S} & \mathrm{S} & \mathrm{S} & \mathrm{S} & \mathrm{S}\end{array}$

fermentum $\mathrm{N} 13$

$L$.

S S

S

S

S

S S

S

plantarumN14
L. case $i \mathrm{~N} 15$

S

S

S

S

S

S S

S

L. brevis $\mathrm{N} 10$

S

S

S

S

S

S

S

S

Keys: The range according to CSLI, 2000., $\mathrm{R}=$ Resistance, $\mathrm{S}=$ Sensitive

\subsection{CONCLUSIONS}

The results showed that LAB strains such as Lactobacillus casei 1 1, L.brevisN10, L. plantarum $\mathrm{N} 24$, and L. plantarum $\mathrm{N} 17$ from fermented milk product (nono) had maximum antimicrobial activity, survived acidic $\mathrm{pH}$ and bile, sensitive to various antibiotics, and safe indicating they are suitable probiotic candidates which can be used as food supplements. 


\section{ACKNOWLEGDEMENTS}

I would like to express my gratitude to Professor A. A. Onilude, Department of Microbiology, University of Ibadan, Nigeria for his valueable supervision and guidance. I also have to thank Dr.Wakil for her counsel and guidance.

\section{REFERENCES}

1. Adebayo-tayo, B. C. and Onilude, A. A. 2008.Screening of Lactic Acid Bacteria Strains Isolated from Some Nigerian Fermented Foods for EPS Production. World Applied Sciences Journal 4: 741-747.

2. Ahmed, T. and Kanwal, R. 2004. Biochemical characteristics of lactic acid producing bacteria and preparation of camel milk cheese by using starter culture. Pakistan Veterinairy Journal 24:87-91.

3. Bauer, A.W., Kirby, W. M. M. and Sheris, J. C. 1996. Antibiotic susceptibility testing by a standardized single disk method. American Journal of Clinical Pathology 45: 493-496.

4. Brant, R. J. and Todd, R. K. 2014. Impact of genomics on the field of probiotic research: historical perspectives to modern paradigms. A. V. Leeuwenhoek 106:141-156..

5. Gupta, H. and Malik, R.K 2007. Incidence of virulence in bacteriocin producing enterococcal isolates. Lait87:587-601

6. Handa, S. and Sharma, N. 2016. In vitro study of probiotic properties of Lactobacillus plantarum F22 isolated from Chhang-A traditional fermented beverage of Himachal Pradesh, India. Journal of Genetic Engineering and Biotechnology14(1): 91-97.

7. Hasegawa, T., Minami, M., Okamato, A., Tatsuno, I., Isaka, M. and Ohta, M. 2010.Characterisation of a virulence- associated and cell wall located DNase of Streptococcus pyogenes. Microbiology 186:184-190

8. Hoque, M. Z., Akter, F., Hossain, K. M., Rahman, M. S. M., Billah, M. M. and Islam, K. M. D. 2010. Isolation, Identification and Analysis of Probiotic Properties of Lactobacillus Spp.From Selective Regional Yoghurts. World Journal of Dairy and Food Sciences5 (1): 39-46.

9. Kabore, D., Sawadogo-Lingani, H., Dicko, M.H., Diawara, B. and Jakobsen, M. 2012. Acid resistance, bile tolerance and antimicrobial properties of dominant lactic acid bacteria isolated from traditional maari" baobab seeds fermented condiment. African Journal of Biotechnology11:1197-1206.

10. Klingberg, T., Axelsson, K., Naterstad, D., Elsser and Budde, B.B. 2005. Identification of potential probiotic starter cultures for Scandinavian-type fermented sausages. International Journal of Food Microbiology105:419-431.

11. Kos, B., Suskovic, J, Vukovic, S, Simpraga, M, Frece, J, Matosic, S. 2003. Adhesion and aggregation ability of probiotic strain Lactobacillus acidophilus M92. Journal of Applied Microbiology 94:981-987.

12. Krishnendra, S., Nama, S. D., Priyanka, P., Priyanka, S., Neelofar, S. and Jitendra, N. 2013. Antagonistic Activity of Lactic Acid Bacteria from Dairy Products. International Journal of Pure and Applied Bioscience $1(1): 28-32$.

13. Kumar, A. and Kumar, D. 2015. Characterization of Lactobacillus isolated from dairy samples for probiotic properties. Anaerobe33: 117-123.

14. Masci, E. 2013. Bacteria and intestinal health in adult and pediatric population: moving from the field of alternative medicine to evidence-based treatment. International Journal of Probiotics and Prebiotics 8: 1-4

15. Martin, R., Olivares, M., Marin, M. L. and Fernandez, L. 2005. Probiotic potential of 3 Lactobacillus strains isolated from breast milk. Journal of Human Lactation 21:8-17.

16. Mishra,V. and Prasad, M. N. 2005. Application of in vitro for selection of Lactobacillus casei strains as potencial probiotics. International Journal of Food Microbiology 103:109-115.

17. Mohammed, S., Ahlgren, J. A. and Horne, D. 2016. Structural characterization and biological activities of an exopolysaccharide kefi ran produced by Lactobacillus kefiranofaciens WT-2B(T). Journal of Agricultural and Food Chemistry 52: 5533-5538.

18. Nikolic, M., Terzic-Vidojevic, A., Jovcic, B., Begovic, Golic, N. and Topisirovic, L. 2008. Characterization of lactic acid bacteria isolated from Bukuljac, a homemade goat's milk cheese. International Journal of Food Microbiology 122:162-170. 
19. Noruga, L., Cuevas, I., Margolles, A. and de los Reyes, C. 2006.Deconjugation and bile salts hydrolase activity by Bifidobacterium strain with acquired resistance to bile. International Dairy Journal 16: 850-855.

20. Orlowaski, A. and Bielecka, M. 2006. Preliminary characteristics of Lactobacillus and Bifidobacteriumstrains as probiotic candidates. Poland Journal of Food Nutrition and Science 15:269-276.

21. Nwanyanwu, C. E., Alisi, C. S., Nweke, C. O. and Orji, J. C. 2012. Cell surface properties of phenolutilizing bacteria isolated from petroleum refinery wastewater. Journal of Research Biology 2:383-391.

22. Pérez-Chabela, M. L., Díaz-Vela, J., Reyes-Menéndez, C.V. and Totosaus, A. 2013. Improvement of moisture stability and textural properties of fat and salt reduced cooked sausages by inoculation of thermotolerant lactic acid bacteria. International Journal of Food Properties.

23. Pérez-Chabela, M.L., Lara-Labastida, R., Rodríguez-Huezo, M.E. and Totosaus, A. 2012.Effect of spray drying encapsulation of thermotolerant lactic acid bacteria on meat batters properties. Food Bioprocess and Technology7:45-98 DOI: 10.1007/s11947-012-0865-y.

24. Pérez-Chabela, M.L., Totosaus, A. and Guerrero, I. 2008. Evaluation of thermotolerant capacity of lactic acid bacteria isolated from commercial sausages and the effects of their inoculation on the quality of cooked sausages. Ciência e Tecnologia de Alimentos, Campiñas,28: 132-138..

25. Rivera-Espinoza, Y. and Gallardo-Navarro, Y. 2010.Non-dairy probiotic products.Food Microbiology27:1-11.

26. Rosenberg, M., Gutnic, G. L. and Rosenberg, E. 2013. Adherence of bacteria to hydrocarbons: a simple method for measuring cell-surface hydrophobicity. Microbiology Letters 9:29-33

27. Yadesse, G., Ephraim, E. and Ashenafi, M. 2005 Assessment of the antimicrobial activity of lactic acid bacteria isolated from Borde and Shamita, traditional Ethiopian fermented beverages, on some food-borne pathogens and effect of growth medium on the inhibitory activity. The International Journal of Food Safety 5: $13-20$

28. Tambekar, D. H., Bhutada, S. A., Choudhary, S. D. and Khond, M. D. 2009. Assessment of potential probiotic bacteria isolated from milk of domestic animals. Journal of Applied Bioscience15: 815-819.

29. Tambekar, D. H. and Bhutada, S. A. 2010. Studies on antimicrobial activity and characteristics of bacteriocins produced by Lactobacillus strains,isolated from milk of domestic animals. The International Journal of Microbiology 8: 1-6.

30. Vasiee, A. R., Tabatabaei, M., Yazdi, F., Mortazavi, A. and Edalatian, M. R. 2014.Isolation, identification and characterization of probiotic Lactobacilli spp. from Tarkhineh. International Food Research Journal 21(6): 2487-2492. 